\title{
Dynamic Responses of Rotor Drops onto Auxiliary Bearing with the Support of Metal Rubber Ring
}

\author{
Zhu Yili* and Zhang Yongchun
}

Department of Electrical Engineering, Changzhou Institute of Technology, Changzhou, Jiangsu, 213002, P.R. China

\begin{abstract}
In an active magnetic bearing (AMB) system, the Auxiliary bearings (ABs) are indispensable to protect the rotor and stator in case of $\mathrm{AMB}$ failure. Most of the former researches try to modify relevant design parameters of ABs to buffer the following impacts and heating after rotor drop. Based on the analysis of the disadvantages of traditional ABs, a new type of $\mathrm{AB}$ with the support of metal rubber ring is proposed to enhance the $\mathrm{AB}$ work performance in $\mathrm{AMB}$ system. Detailed simulation models containing rigid rotor model, contact model between rotor and inner race as well as $\mathrm{AB}$ system model after rotor drop are established. Then, using those established models the dynamic responses are simulated to obtain proper metal rubber ring support characteristics. Finally, relevant rotor drop experiments are carried out on the established AMB test bench. The experiment results verify the advantages of the new type ABs and the correctness of simulation analysis.
\end{abstract}

Keywords: Active magnetic bearing, auxiliary bearing, dynamic response, metal rubber ring, rotor drop.

\section{INTRODUCTION}

Active magnetic bearings (AMBs) have many advantages over conventional mechanical bearings, such as no mechanical friction and lubrication, adjusted support stiffness and damping. However, the ABs are indispensable to protect the AMB assembly after a possible AMB failure.

Most of the former researches have focused on the dynamic responses after rotor drop. Kirk et al. [1,2] studied the influences of the support stiffness and damping by evaluating dynamic response for various rotor-support system parameters. They showed an optimum damping can be selected to prevent destructive backward whirl. Fumagalli et al. [3] classified the rotor drop process into four distinct motion phases: free fall; impact; sliding; and rolling. Sun [4] conducted numerical simulations of a rotor drop onto the $\mathrm{AB}$ in flywheel energy storage system using a detailed $A B$ model which includes a Hertzian load-deflection relationship between mechanical contacts, speed-and-preload-dependent bearing stiffness due to centrifugal force, and a drag friction torque. Xie and Flowers [5] numerically investigated the steady-state behavior of a rotor drops onto $\mathrm{ABs}$ and primarily looked at the effects of various parametric configurations: rotor imbalance, support stiffness and damping. Foiles and Allaire [6] also numerically analyzed the effects of parameters for non-linear models on two types of rotors: generator or turbine rotor and centrifugal compressor rotor. Cole et al. [7] developed a deep groove $\mathrm{AB}$ model considering the elastic deformation of the inner race, mainly studied the impact force and effects of bearing width as well as ball load distributions. Kaur [8] tested the performances of powder lubricated bearings used as AB.
However, most of those researches focused on the relevant parameters of $A B$ itself. For it is hard to modify the bearing stiffness and damping to satisfy the optimum simulation results, a new type $\mathrm{CB}$ with the support of metal rubber ring is proposed in this paper. Because of the excellent buffer characteristics of metal rubber ring, it can to some extent buffer rotor vibrations after rotor drop. The performances of the new type $\mathrm{AB}$ are analyzed by both simulations and experiments.

\section{SIMULATION}

\subsection{Structure of AMB System}

Fig. (1) shows the studied structure of a motor drive system equipped with magnetic bearings, where 1: rotor; 2 : radial displacement sensor; 3: axial displacement sensor; 4: auxiliary bearing; 5: axial magnetic bearing; 6: radial magnetic bearing; 7-motor. The motor is located between the

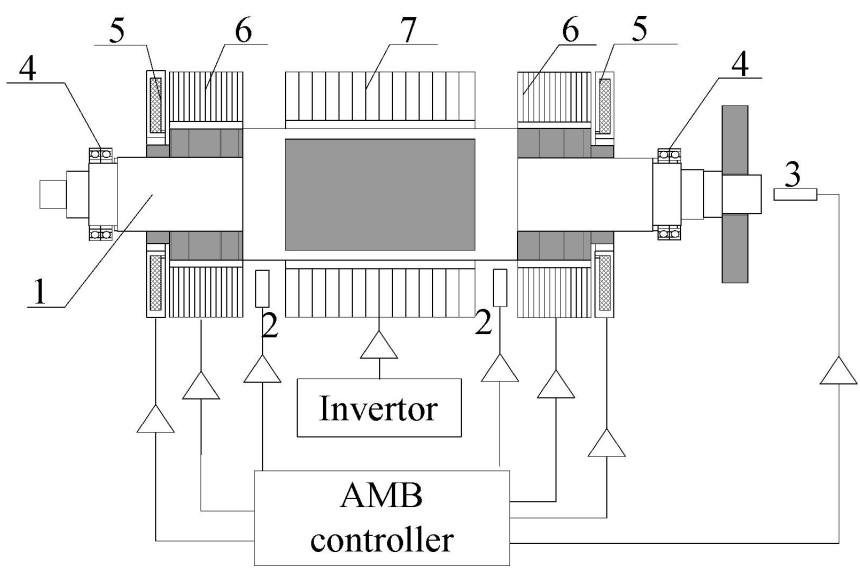

Fig. (1). Structure of AMB System. 
two radial magnetic bearings. Each radial magnetic bearing generates radial forces. Axial magnetic bearings regulate the axial forces in the shaft direction. Besides those magnetic bearings, two auxiliary bearings are located in the two ends of the structure respectively to prevent damages after rotor drop. The air gap between the auxiliary bearing inner race and the rotor is half of the air gap of AMB.

The two types of AB are presented in Fig. (2). Compared with traditional $\mathrm{AB}$, a metal rubber ring is installed in the new type $A B$.

(a) Traditional auxiliary bearing

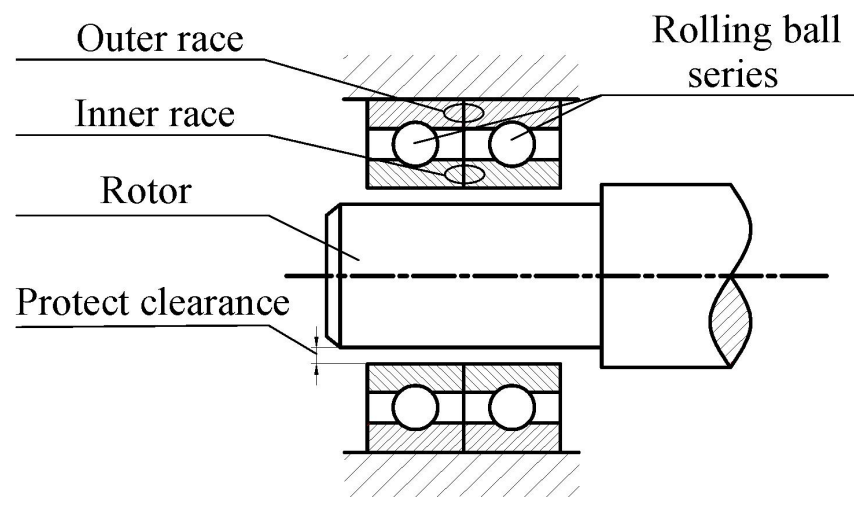

(b) New type auxiliary bearing

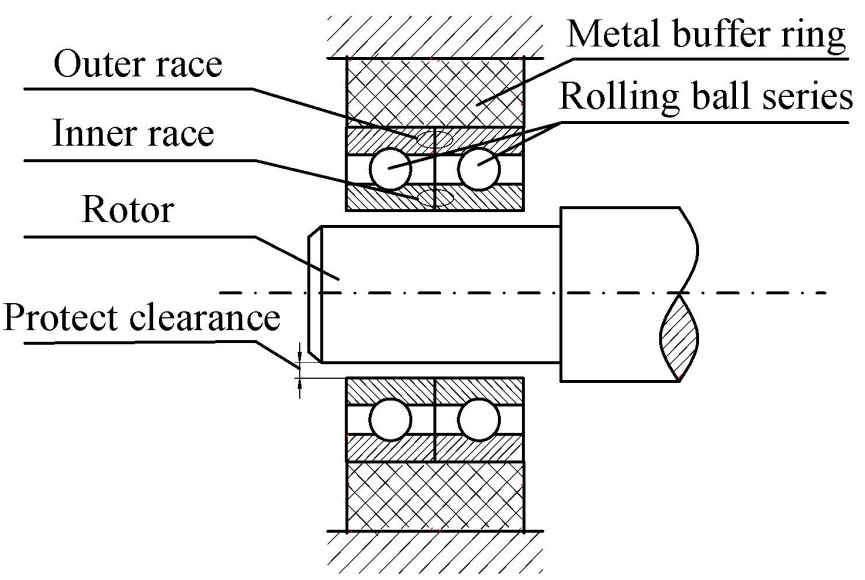

Fig. (2). Structure of analyzed auxiliary bearing.

\subsection{Simulation Model}

According to the structure of AMB system, the rotor force model can be obtained, as shown in Fig. (3). When the AMB system is in normal operation, the rotor bears left-hand and right-hand magnetic bearing forces $\left(F_{\mathrm{a} 1 \mathrm{x}}, F_{\mathrm{a} 1 \mathrm{y}}\right.$ and $F_{\mathrm{a} 2 \mathrm{x}}$, $\left.F_{\text {a2y }}\right)$, centrifugal forces $\left(F_{\text {cx }}, F_{\text {cy }}\right)$ and gravity $\left(G_{\mathrm{r}}\right)$ respectively. After AMB system failure, the rotor bears lefthand and right-hand $\mathrm{AB}$ forces $\left(F_{\mathrm{b} 1 \mathrm{x}}, F_{\mathrm{b} 1 \mathrm{y}}\right.$ and $\left.F_{\mathrm{b} 2 \mathrm{x}}, F_{\mathrm{b} 2 \mathrm{y}}\right)$, centrifugal forces $\left(F_{\mathrm{cx}}, F_{\mathrm{cy}}\right)$ and gravity $\left(G_{\mathrm{r}}\right)$ respectively.

Then the rotor motion equation can be written as:

$m \ddot{x}+G \dot{x}=A F_{a}+B_{b}+F_{c}+F_{g}$

where mass matrix $\mathrm{m}=\operatorname{diag}\left(m_{\mathrm{r}}, m_{\mathrm{r}}, J, J\right), m_{\mathrm{r}}$ is rotor mass, $J$ is rotor transverse moment of inertia (MOI), barycenter displacement $\mathrm{x}=\left(x_{\mathrm{r}}, y_{\mathrm{r}}, \theta_{x}, \theta_{y}\right)^{\mathrm{T}}, \quad x_{\mathrm{r}}$ and $y_{\mathrm{r}}$ are the displacements of barycenter in the direction of $x$ and $y$ axis respectively, $\theta_{\mathrm{x}}$ and $\theta_{\mathrm{y}}$ are the rotational displacement of barycenter around the direction of $x$ and $y$ axis respectively. $G$ is the gyroscopic torque matrix,

$\mathrm{G}=\left(\begin{array}{cccc}0 & 0 & 0 & 0 \\ 0 & 0 & 0 & 0 \\ 0 & 0 & 0 & \omega J_{z} \\ 0 & 0 & -\omega J_{z} & 0\end{array}\right)$

$J_{\mathrm{z}}$ is rotor polar MOI, $\omega$ is the rotor angular velocity. A and $\mathrm{B}$ are the introduced coefficient matrixes,

$\mathrm{A}=\left(\begin{array}{cccc}1 & 0 & 1 & 0 \\ 0 & 1 & 0 & 1 \\ 0 & l_{\mathrm{a} 1} & 0 & -l_{\mathrm{a} 2} \\ -l_{\mathrm{a} 1} & 0 & l_{\mathrm{a} 2} & 0\end{array}\right), \mathrm{B}=\left(\begin{array}{cccc}1 & 0 & 1 & 0 \\ 0 & 1 & 0 & 1 \\ 0 & l_{\mathrm{b} 1} & 0 & -l_{\mathrm{b} 2} \\ -l_{\mathrm{b} 1} & 0 & l_{\mathrm{b} 2} & 0\end{array}\right)$

magnetic force vector $\mathrm{F}_{\mathrm{a}}=\left(F_{\mathrm{a} 1 x}, F_{\mathrm{a} 1 y}, F_{\mathrm{a} 2 x}, F_{\mathrm{a} 2 y}\right)^{\mathrm{T}} ;$ rotor centrifugal force vector $\mathrm{F}_{\mathrm{a}}=\left(F_{c x}, F_{c y}, 0,0\right)^{\mathrm{T}}$; rotor gravity vector $\mathrm{F}_{g}=\left(F_{g x}, F_{g y}, 0,0\right)^{\mathrm{T}}$.

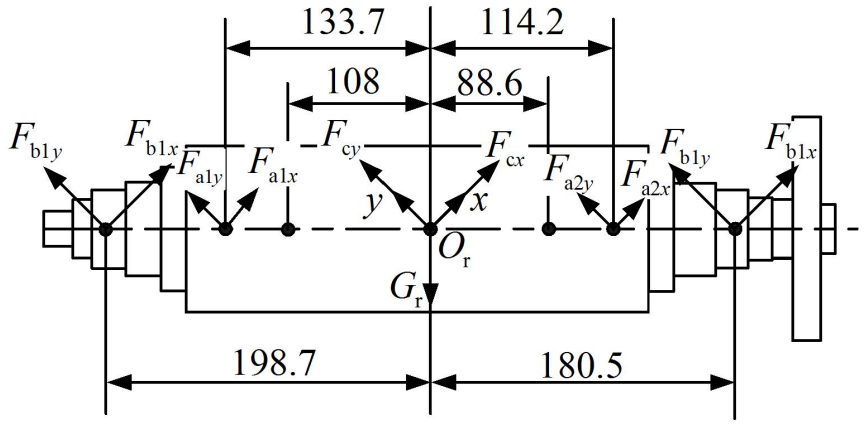

Fig. (3). Rotor force model.

The contact model after rotor drops onto the $\mathrm{AB}$ supported by metal rubber ring is shown in Fig. (4). Here the model of metal rubber ring is seemed as stiffness $K_{\mathrm{rr}}$ and damping $C_{\mathrm{rr}}$, which is installed between the bearing house and foundation support. $F_{\mathrm{n}}$ and $F_{\mathrm{t}}$ are the normal impact force and tangential frictional force between the rotor and inner race, respectively; $R_{\mathrm{r}}$ and $R_{\mathrm{b}}$ are the shaft radius and inner race bore radius at the $\mathrm{AB}$ position, respectively; $m_{\mathrm{i}}$ is the mass of the inner race, and the bearing outer race is rigidly installed in the bearing house, the whole mass is $m_{\mathrm{o}}$; $C_{\mathrm{b}}$ and $C_{\mathrm{rr}}$ are the support damping of the bearing and metal rubber ring, respectively; $x_{\mathrm{b}}$ and $x_{\mathrm{i}}$ are the vibration displacements of the rotor and inner race in the $x$-axis, respectively; $y_{\mathrm{b}}$ and $y_{\mathrm{i}}$ are the vibration displacements of the rotor and inner race in the $y$-axis, respectively; $\phi_{\mathrm{i}}$ is the defined rotor-inner race contact angle, and $\sin \phi_{\mathrm{i}}=\left(y_{\mathrm{i}}-y_{\mathrm{o}}\right) / u_{\mathrm{i}}$, $\cos \phi_{\mathrm{i}}=\left(x_{\mathrm{i}}-x_{\mathrm{o}}\right) / u_{\mathrm{i}}$, where $u_{\mathrm{i}}=\sqrt{\left(x_{\mathrm{i}}-x_{\mathrm{o}}\right)^{2}+\left(y_{\mathrm{i}}-y_{\mathrm{o}}\right)^{2}}$, To simplify the analysis, it is assumed that there always exists a ball labeled as " 1 " in the direction of relative displacement 
between the inner and outer race, so the $j$ th ball position angle moving counterclockwise is $\varphi_{j}=2 \pi(j-1) / N_{\mathrm{b}}$, where $N_{\mathrm{b}}$ is the ball number of the single bearing. In the same way, $\theta_{\mathrm{r}}$ and $\theta_{\mathrm{i}}$ are the angular displacements of the rotor and inner race respectively, and $\delta_{\mathrm{b}}$ is the penetration depth between the rotor and inner race.

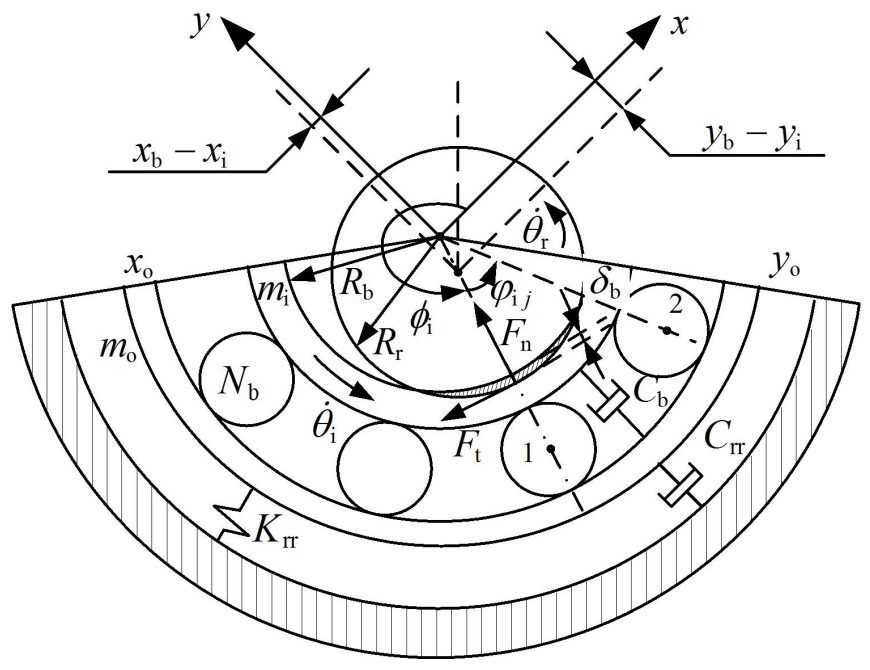

Fig. (4). Contact model after rotor drop.

The radial motion equation for the new type $A B$ inner race and bearing house after rotor drop is given by

$\mathrm{M}_{\mathrm{b}} \ddot{\mathrm{x}}_{\mathrm{b}}+\mathrm{C}_{\mathrm{b}} \dot{\mathrm{x}}_{\mathrm{b}}=\mathrm{F}_{\mathrm{b}}+\mathrm{F}_{\mathrm{rb}}$

where the mass matrix $\mathrm{M}_{\mathrm{b}}=\operatorname{diag}\left(m_{\mathrm{i}}, m_{\mathrm{i}}, m_{\mathrm{o}}, m_{\mathrm{o}}\right)$; the forces from the balls and metal rubber ring $\mathrm{F}_{\mathrm{b}}=\left(F_{\mathrm{i} x}, F_{\mathrm{i} y}, F_{\mathrm{e} x}+F_{r x}, F_{\mathrm{e} y}+F_{r y}\right)^{\mathrm{T}}$; the vibration displacement vector $\mathrm{x}_{\mathrm{b}}=\left(x_{\mathrm{i}}, y_{\mathrm{i}}, x_{\mathrm{o}}, y_{\mathrm{o}}\right)^{\mathrm{T}}$; the forces from the rotor $\mathrm{F}_{\mathrm{rb}}=\left(F_{\mathrm{b} x}, F_{\mathrm{b} y}, 0,0\right)^{\mathrm{T}}$; the damping matrix

$\mathrm{C}_{\mathrm{b}}=\left[\begin{array}{cccc}2 C_{\mathrm{b}} & 0 & -2 C_{\mathrm{b}} & 0 \\ 0 & 2 C_{\mathrm{b}} & 0 & -2 C_{\mathrm{b}} \\ -2 C_{\mathrm{b}} & 0 & 2 C_{\mathrm{b}}+C_{\mathrm{rr}} & 0 \\ 0 & -2 C_{\mathrm{b}} & 0 & 2 C_{\mathrm{b}}+C_{\mathrm{rr}}\end{array}\right]$.

The rotational equation of the inner race can be written as:

$J_{z} \ddot{\theta}_{\mathrm{i}}=F_{\mathrm{t}} R_{\mathrm{b}}-2 T_{\mathrm{i}}$

where $T_{\mathrm{i}}$ is the internal friction moment of the bearing.

\subsection{Simulation Results}

The whole simulation composes two parts, rotor motions before and after AMB failure. Firstly, the rotor motions during normal operation are simulated as the initial conditions for the dynamic simulations after rotor drop. During the simulation, based on relevant AMB theory, hertz contact theory and well as ball bearing support theory [9], the $\mathrm{AMB}$ forces and $\mathrm{AB}$ forces are calculated using the real time rotor and $\mathrm{AB}$ motions. The chosen ball bearing type is 61905 , and some other simulation parameters are listed in Table 1.

Table 1. Relevant simulation parameters.

\begin{tabular}{|c|c|}
\hline Parameter & Value \\
\hline \hline Rotor unbalance $e_{\mathrm{r}}(\mu \mathrm{m})$ & 2.5 \\
\hline Rotor transverse MOI $J\left(\mathrm{~kg} \cdot \mathrm{mm}^{2}\right)$ & $6.1 \mathrm{e} 5$ \\
\hline Rotor polar MOI $J_{\mathrm{z}}(\mathrm{kg} \cdot \mathrm{mm} 2)$ & $4.7 \mathrm{e} 3$ \\
\hline Mass of the inner race $m_{\mathrm{i}}(\mathrm{kg})$ & $16.4 \mathrm{e}-3$ \\
\hline Mass of the bearing house $m_{\mathrm{o}}(\mathrm{kg})$ & 0.35 \\
\hline Current stiffness of AMB $k_{\mathrm{i}}(\mathrm{N} / \mathrm{A})$ & 166.5 \\
\hline Displacement stiffness of AMB $k_{\mathrm{x}}(\mathrm{N} / \mathrm{m})$ & $1.25 \mathrm{e} 6$ \\
\hline Protective gap of ABs $c_{\mathrm{r} 1}(\mathrm{~mm})$ & 0.125 \\
\hline Mass of rotor $m_{\mathrm{r}} \mathrm{kg}$ & 9.1 \\
\hline
\end{tabular}

The maximum impact forces between rotor and inner race after rotor drop for different metal rubber ring support stiffness and damping at the initial rotor rotational speed 12 $000 \mathrm{r} / \mathrm{min}, 18000 \mathrm{r} / \mathrm{min}, 24000 \mathrm{r} / \mathrm{min}$ and $30000 \mathrm{r} / \mathrm{min}$ can be calculated after obtaining the rotor and $\mathrm{AB}$ motions by simulation. The results are presented in Fig. (5). According to the simulation results, it can be seen that choosing support stiffness $1 \mathrm{e} 6 \mathrm{~N} / \mathrm{m}$ and support damping $1 \mathrm{e} 4 \mathrm{~N} . \mathrm{s} / \mathrm{m}$ are advisable to buffer the following impact forces after rotor drop.

\section{EXPERIMENTS}

Providing the above obtained support stiffness and damping, the metal rubber ring is manufactured by professional factory. Fig. (6) shows the part installed the metal rubber ring. Fig. (7) presents the rotor drop test rig, where 1:PC; 2: AMB system controller; 3: high-speed magnetic levitation motor; 4: inverter; 5: Labview data acquisition (DAQ) boards; 6: software of data acquisition system. The displacement sensor signals are collected by Labview DAQ boards and saved in the PC. A subsequent analysis of the collected data is carried out using MATLAB software.

Using the collected displacement sensor signals, the rotor orbits $0.1 \mathrm{~s}$ before rotor drop and $0.2 \mathrm{~s}$ after rotor drop onto different types of $A B$ at the initial rotor speed $12000 \mathrm{r} / \mathrm{min}$ can be obtained, as shown in Fig. (8). It is obviously that the use of metal rubber ring can effectively reduce the vibrations after rotor drop.

Once the rotor vibrations after rotor drop are obtained by the experiments, using equation (1) the support forces of ABs can be calculated as:

$\mathrm{F}_{\mathrm{b}}=\operatorname{inv}\left(\mathrm{m} \ddot{\mathrm{x}}+\mathrm{G} \dot{\mathrm{x}}-\mathrm{F}_{\mathrm{c}}-\mathrm{F}_{\mathrm{g}}\right)$

The maximum impact forces calculated using the experimental results $0.5 \mathrm{~s}$ after rotor drops are shown in Fig. (9). The results indicate that the proposed new type $\mathrm{AB}$ can effectively reduce the impact forces after rotor drop. 
(a) $12000 \mathrm{r} / \mathrm{min}$

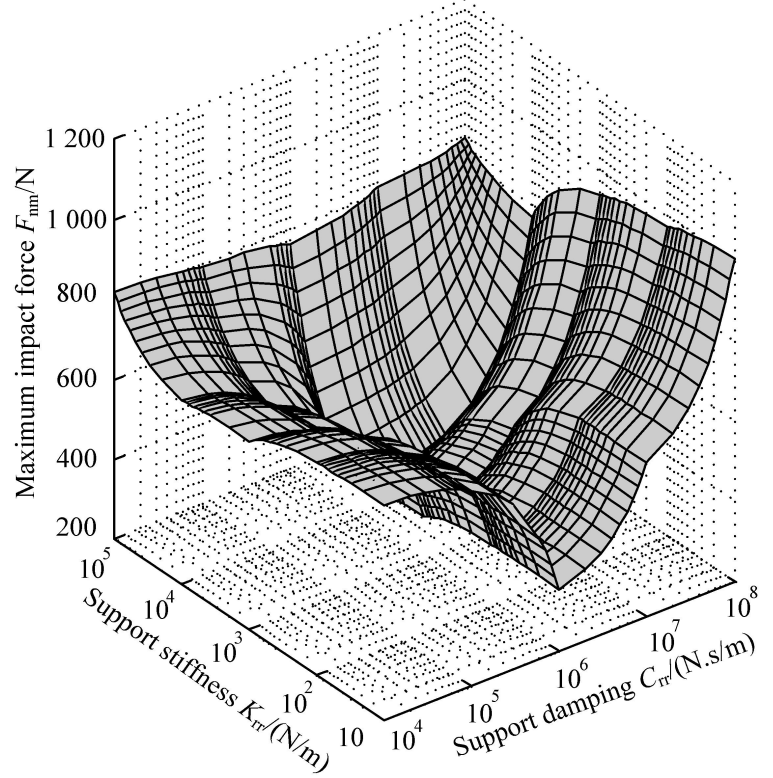

(c) $24000 \mathrm{r} / \mathrm{min}$

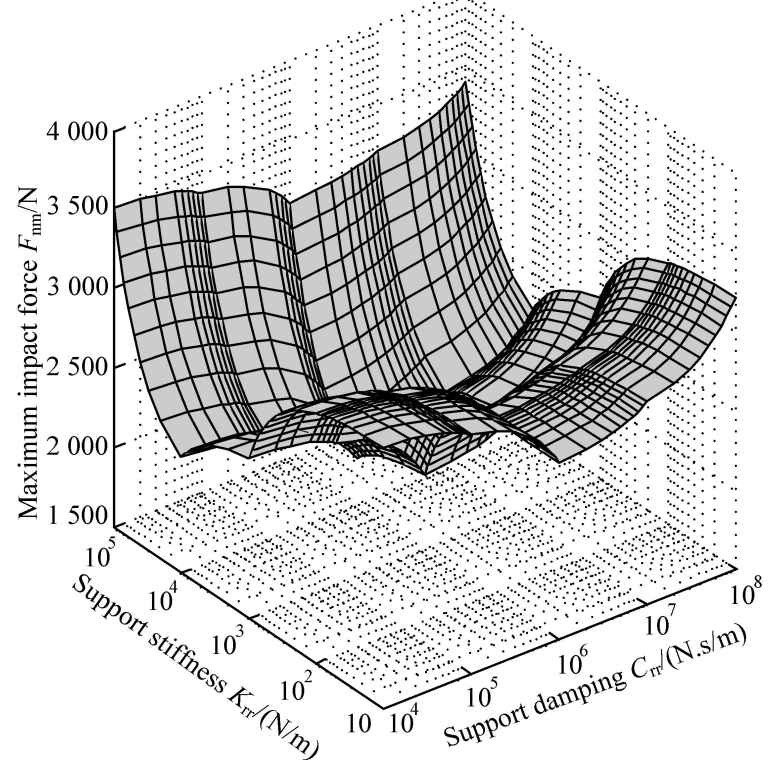

(b) $18000 \mathrm{r} / \mathrm{min}$

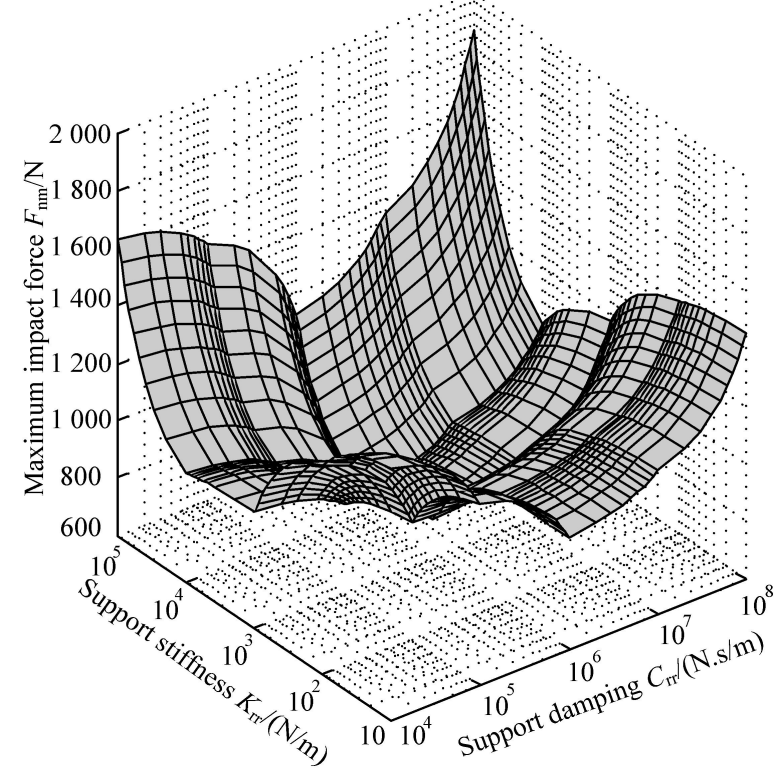

(d) $30000 \mathrm{r} / \mathrm{min}$

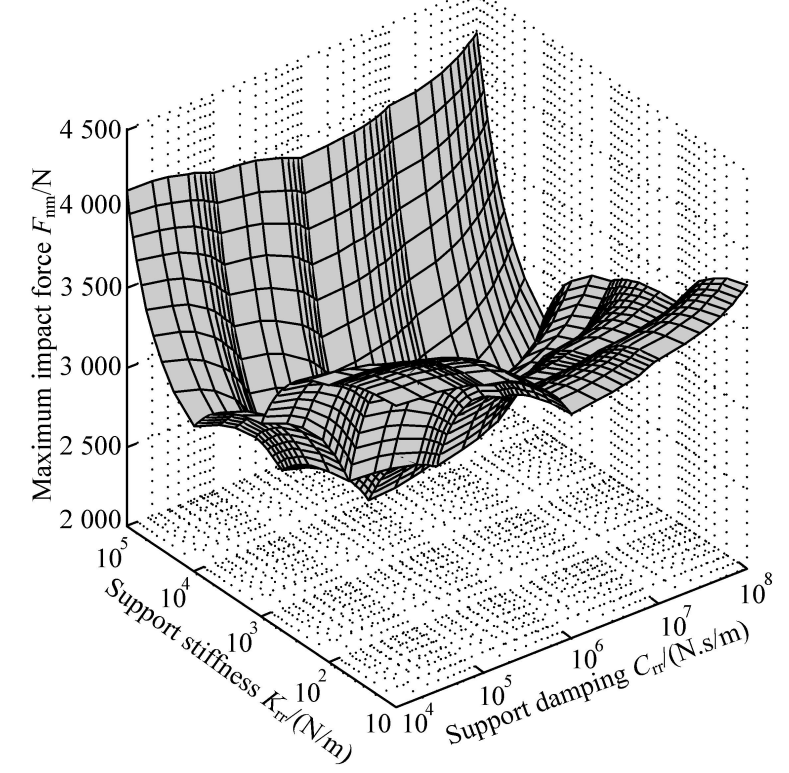

Fig. (5). Influences of metal rubber ring support characteristics on maximum impact forces.

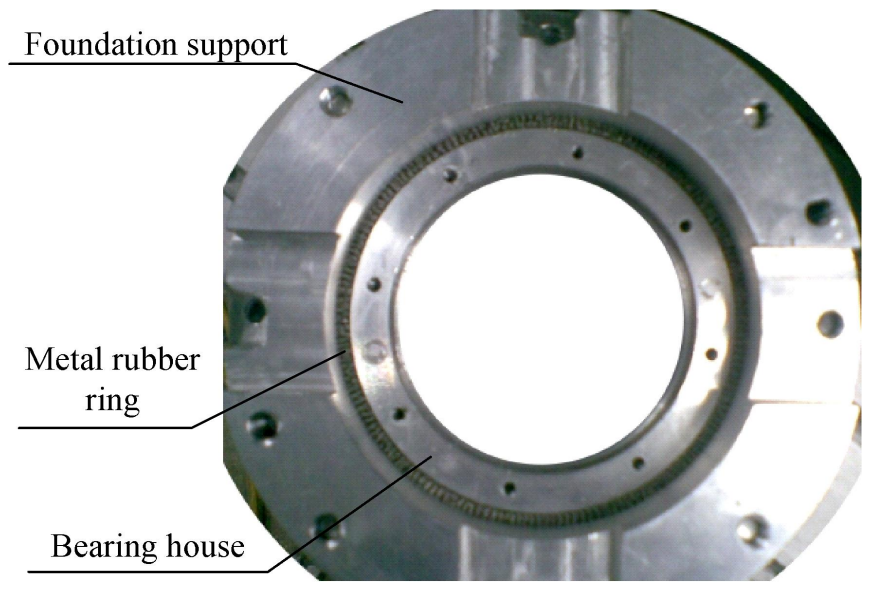

Fig. (6). Photograph of new type AB block.

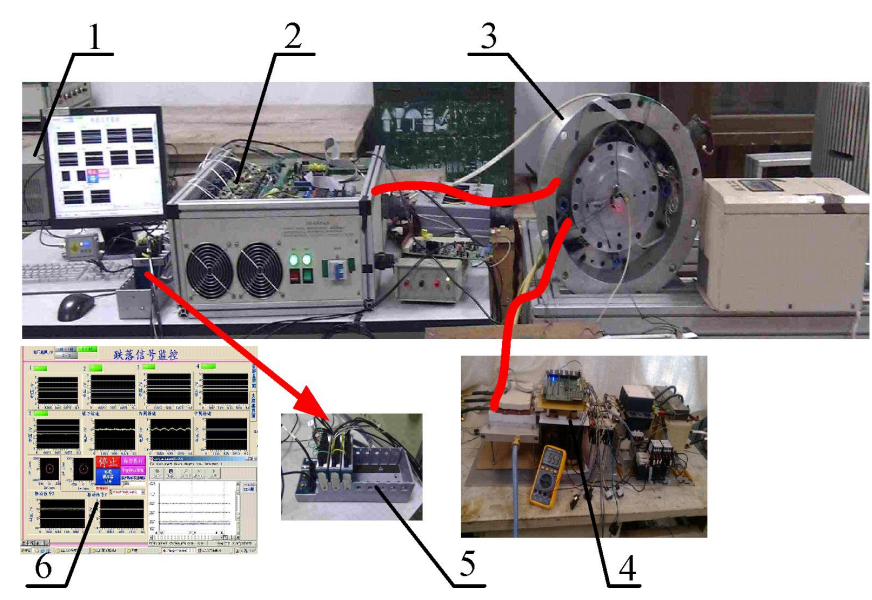

Fig. (7). Rotor drop experiment rig. 
(a) Traditional $\mathrm{AB}$

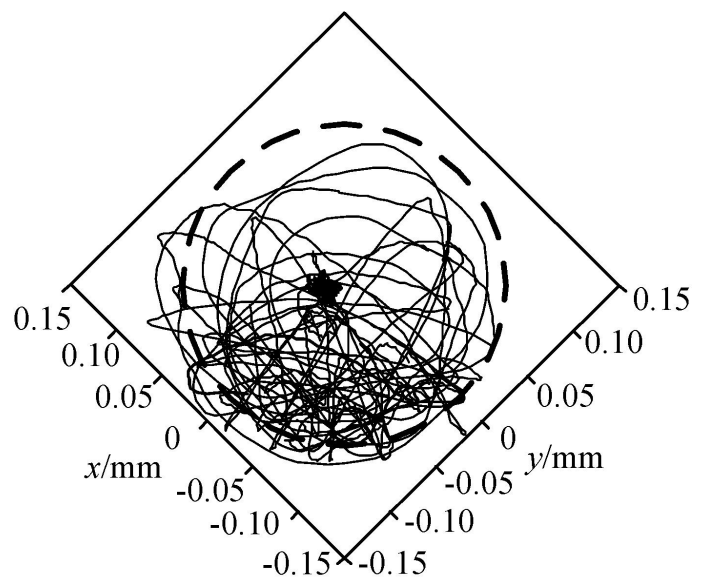

(b) New type $\mathrm{AB}$

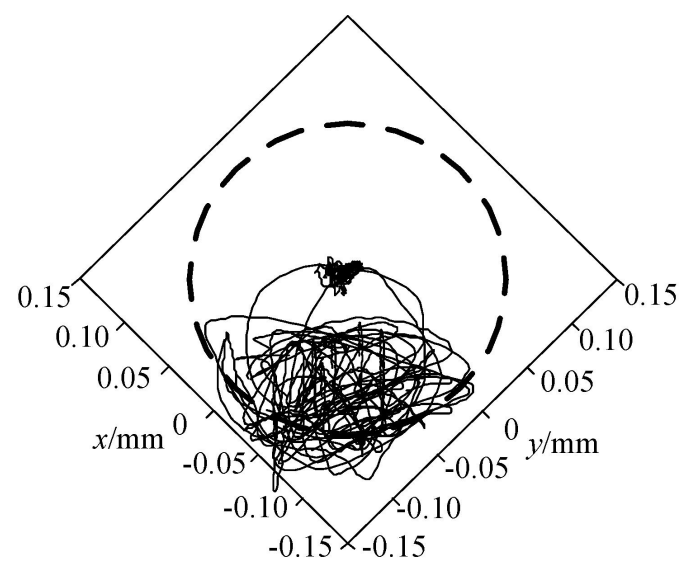

Fig. (8). Rotor orbits obtained by experiments.

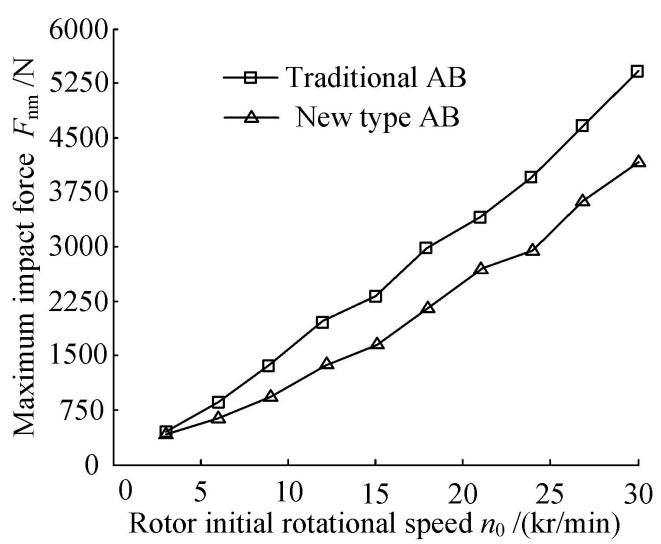

Fig. (9). Maximum impact force obtained by experiments.

\section{CONCLUSION}

In this paper, the new type $\mathrm{AB}$ with metal rubber ring is proposed to improve the working performances of $A B$ in AMB system. The relevant dynamic responses after AMB failure are theoretically simulated and experimentally verified. The following conclusions can be obtained from the above researches.

1) It is necessary to establish dynamic models to obtain proper support characteristics of metal rubber ring before manufacture.

2) Use of proper metal rubber ring helps to reduce the rotor vibration amplitudes and impact forces after rotor drop.

\section{CONFLICT OF INTEREST}

The authors confirm that this article content has no conflict of interest.

\section{ACKNOWLEDGEMENTS}

This work was financially supported by National Natural Science Foundation of China (51405040); the Applied Basic Research in Chanzhou city of China (CJ20140048).

\section{REFERENCES}

[1] T. Ishii, and R. G. Kirk, "Transient response technique applied to active magnetic bearing machinery during rotor drop," Journal of Rotating Machinery and Vehicle Dynamics, vol. 35, pp. 191-199, 1991.

[2] R. G. Kirk, and T. Ishii, "Transient rotor drop analysis of rotor following magnetic bearing power outage," In: Proceedings of MAG '93 Magnetic Bearings, Magnetic Drives, and Dry Gas Seals Conference \& Exhibition, Alexandria, VA, USA, 1993, pp. 53-61.

[3] M. Fumagalli, P. Varadi, and G. Schweitzer, "Impact dynamics of high speed rotors in retainer bearings and measurement concepts," In: Proceedings of $4^{\text {th }}$ International Symposium on Magnetic Bearings, Zurich, Switzerland, pp. 239-244, 1994.

[4] G. Sun, A. B. Palazzolo, A. Provenza, and G. Montague, "Detailed ball bearing model for magnetic suspension auxiliary service," Journal of Sound and Vibration, vol. 269, pp. 933-963, 2004.

[5] H. Xie, and G. T. Flowers, "Steady-state dynamic behavior of an auxiliary bearing supported rotor system," In: Proceedings of American Society of Mechanical Engineers Winter Annual Meeting, Chicago, USA, 1994, pp. 1-11.

[6] W. C. Foiles, and P. E. Allaire, "Nonlinear transient modeling of active magnetic bearing rotors during rotor drop on auxiliary bearing," In: Proceedings of $M A G$ ' 97 Industrial Conference and Exhibition on Magnetic Bearings, Alexandria, VA, USA, 1997, pp. 154-163.

[7] M. O. T. Cole, P. S. Keogh, and C. R. Burrows, "The dynamic behavior of a rolling element auxiliary bearing following rotor impact", Journal of Tribology, vol. 124, pp. 406-606, 2002.

[8] R. G. Kaur, and H. Heshmat, "100 mm diameter self-contained solid/powder lubricated auxiliary bearing operated at 30,000 rpm ", Tribology Transactions, vol. 45, no. 1, pp. 76-84, 2002.

[9] T. A. Harris, Rolling Bearing Analysis, $3^{\text {rd }}$ ed. John Wiley \& Sons Ltd, 1991, pp. 15-45. 